\title{
Prognostic significance of cribriform adenocarcinoma of the lung: validation analysis of 1,057 Japanese patients with resected lung adenocarcinoma and a review of the literature
}

\author{
Naoki Nakajima ${ }^{1}$, Akihiko Yoshizawa ${ }^{1}$, Mariyo Rokutan-Kurata ${ }^{1}$, Misa Noguchi $^{2}$, Yuki Teramoto ${ }^{1}$, \\ Shinji Sumiyoshi ${ }^{1}$, Kyoko Kondo ${ }^{1}$, Makoto Sonobe ${ }^{2}$, Masatsugu Hamaji ${ }^{2}$, Toshi Menju ${ }^{2}$, Hiroshi Date ${ }^{2}$, \\ Hironori Haga ${ }^{1}$ \\ ${ }^{1}$ Department of Diagnostic Pathology, Kyoto University Hospital, Kyoto, Japan; ${ }^{2}$ Department of Thoracic Surgery, Kyoto University Hospital, \\ Kyoto, Japan \\ Contributions: (I) Conception and design: N Nakajima, A Yoshizawa; (II) Administrative support: H Date, H Haga; (III) Provision of study materials \\ or patients: M Noguchi, M Sonobe, M Hamaji, T Menju, H Date; (IV) Collection and assembly of data: N Nakajima, A Yoshizawa, M Rokutan- \\ Kurata, M Noguchi, Y Teramoto, S Sumiyoshi, H Haga; (V) Data analysis and interpretation: N Nakajima, A Yoshizawa; (VI) Manuscript writing: \\ All authors; (VII) Final approval of manuscript: All authors. \\ Correspondence to: Akihiko Yoshizawa, MD, PhD. Department of Diagnostic Pathology, Kyoto University Hospital, 54 Shougoin-Kawaharacho, \\ Sakyo-ku, Kyoto, 606-8507, Japan. Email: akyoshi@kuhp.kyoto-u.ac.jp.
}

Background Cribriform-predominant adenocarcinoma of the lung (Cribri-ADC) is a recently described tumor growth pattern. However, its prognostic impact has not been clearly determined. We analyzed the data of a series of 1,057 Japanese patients with resected lung adenocarcinoma to identify the clinical significance of Cribri-ADC.

Methods: Cribriform pattern (Cribri-p) is defined as invasive back-to-back fused tumor glands with poorly formed glandular spaces or invasive tumor nests comprising tumors cells that produced glandular lumina. We investigated the correlations of Cribri-p and Cribri-ADC with clinicopathological factors as well as diseasefree survival (DFS) and overall survival (OS).

Results: Cribri-p was present in 217 patients (20.5\%) and Cribri-ADC was determined in 25 patients (2.4\%). Cribri-p was associated with larger tumor size, pleural invasion, vascular invasion, lymphatic invasion, and spreading through air spaces (STAS) (all, $\mathrm{P}<0.0001)$. Cribri-ADC was associated with younger age $(\mathrm{P}=0.019)$, vascular invasion $(\mathrm{P}=0.0025)$, STAS $(\mathrm{P}<0.0001)$, and $A L K$ rearrangement $(\mathrm{P}=0.012)$. The DFS curve of patients with Cribri-ADC was identical to that of patients with solid adenocarcinoma; however, the OS curve was located between that of patients with papillary and acinar adenocarcinoma. Of the 10 patients who had tumor recurrences, eight had EGFR mutations or $A L K$ rearrangement, six of whom achieved relatively long survival (median, 64.6, range, 37.4-113 months) following treatment with tyrosine kinase inhibitors (TKIs). In multivariate analysis, Cribri-ADC was not an independent prognostic factor of either recurrence or death.

Conclusions: Cribri-ADC is associated with a higher risk of recurrence; however, most patients can be successfully treated with TKIs.

Keywords: Lung; adenocarcinoma; cribriform; prognosis

Submitted Apr 29, 2020. Accepted for publication Nov 09, 2020.

doi: $10.21037 /$ tlcr-20-612

View this article at: http://dx.doi.org/10.21037/tlcr-20-612 


\section{Introduction}

Lung cancer is the leading cause of cancer-related death worldwide $(1,2)$. Lung adenocarcinoma (L-ADC) is the most common histologic type of lung cancer (3); the latest World Health Organization (WHO) classification for this disease, introduced in 2015 (3), identified five major subtypes according to the most predominant proliferation pattern: lepidic, acinar, papillary, micropapillary, and solid. However, following recent studies, investigators have proposed the cribriform pattern (Cribri-p) as a sixth pattern; cribriform-predominant adenocarcinoma (CribriADC) is reported to be a prognostically unfavorable subtype (4-9). Notably, there were several drawbacks in these studies; the histological definition of Cribri-p was ill-defined, the clinical significance of Cribri-p in other predominant subtypes was not well-examined, and the prognostic significance of Cribri-ADC was described inconsistently (4-9).

To address these shortcomings, we performed this validation study to clearly identify the clinical significance of Cribri-p and Cribri-ADC by reviewing the data of 1057 resected L-ADCs.

We present the following article in accordance with the STROBE reporting checklist (http://dx.doi.org/10.21037/ tlcr-20-612).

\section{Methods}

\section{Cobort}

We retrospectively investigated data of patients with L-ADC who underwent complete curative-intent resection at the Kyoto University Hospital between 2001 and 2015. Patients were excluded from the study if they had multiple primary lung cancers, were treated with chemotherapy or radiotherapy before surgery, underwent incomplete resection, or for whom follow-up information was incomplete based on clinical data retrieved from the Thoracic Surgical Database. The analysis ultimately included 1057 L-ADCs. The requirement for informed consent was waived owing to the retrospective nature of the study and all patients were provided the means to opt out of this study. The study was conducted in accordance with the Declaration of Helsinki (as revised in 2013) and the study protocol was approved by the Kyoto University Hospital ethics committee (R1158-2).

\section{Histological evaluation}

All resected specimens were fixed in formalin, sectioned, and stained with hematoxylin and eosin according to standard procedures. Small-sized tumors were histologically sampled as a whole. Elastic staining was performed to detect pleural or vascular invasion. All of the specimens were reviewed by the authors (NN and AY, both pathologists) who were blinded to the patients' information. The average number of tumor specimens reviewed for each patient was 3.3 (range, 1-20). According to the 2015 WHO classification, each tumor was subjected to comprehensive histological subtyping, and the percentage of each histological component was recorded in $5 \%$ increments (3).

In the current WHO classification, cribriform arrangement was considered as a part of acinar pattern, which is defined as round-to-oval-shaped glands with central luminal space surrounded by tumor cells (Figure 1); however, 'cribriform arrangement' is not clearly defined (3). Furthermore, previous studies described two qualitatively different Cribri-p components; one description is invasive back-to-back fused tumor glands with poorly formed glandular spaces lacking intervening stroma (fused-gland pattern) (Figure 1B,F) or invasive nests of tumors cells that produce glandular lumina without solid components (sievelike pattern) (Figure 1C,G) $(4,6,8,9)$. The other description is nests of tumor cells with sieve-like perforations, which are identical to the 'sieve-like pattern' described by the aforementioned researchers $(5,7)$. In some previous studies, the fused-gland pattern was interpreted under a complex glandular pattern $(5,7)$. In our study, we defined Cribri-p as fused-gland pattern or sieve-like pattern and separately recorded their extents in $5 \%$ increments. Then, we designated tumors as Cribri-p-positive when their proportions of fused-gland and/or sieve-like pattern were $5 \%$ or greater. Cribri-ADC was defined as a tumor which predominantly showed fused-gland and/or sieve-like pattern in the individual tumor (Figure 1). We then evaluated the clinicopathological significance of Cribri-p and Cribri-ADC and studied their prognostic significance.

\section{Detection of genetic alterations in various oncogenes}

To evaluate the association between Cribri-p-exhibiting tumors and gene alterations, such as EGFR, KRAS, 


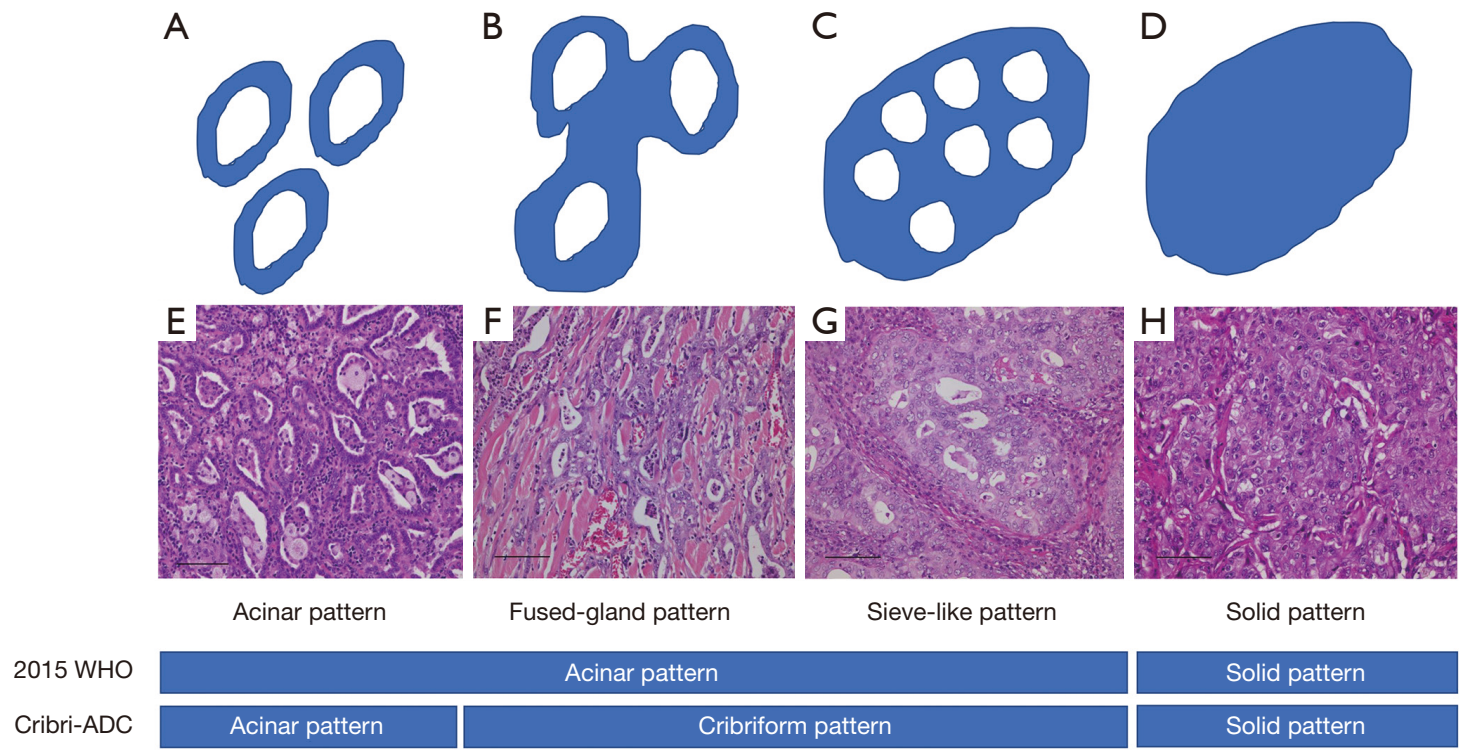

Figure 1 Schema and representative histological images of acinar-, cribriform-, and solid pattern-samples stained with hematoxylin and eosin. (A,E) Acinar pattern showing round-to-oval-shaped glands with a central luminal space surrounded by tumor cells; (B,F) cribriform pattern showing invasive back-to-back fused tumor glands with poorly formed glandular spaces lacking intervening stroma (fused-gland pattern); (C,G) cribriform pattern showing invasive nests of tumors cells that produce glandular lumina without solid components (sievelike pattern); (D,H) solid pattern showing solid nests without lumina. Cribriform adenocarcinoma (Cribri-ADC) was defined as an area of predominantly cribriform pattern including fused-gland and sieve-like pattern within the individual tumor (Cribriform pattern). "2015 WHO" refers to the 2015 World Health Organization classification. Bars indicate $0.1 \mathrm{~mm}$; (E,F,G,H) hematoxylin and eosin staining.

HER2, BRAF, ALK, and ROS1, we used the data that we had performed in our previous studies. Briefly, EGFR mutations were evaluated with the polymerase chain reaction (PCR) single-strand conformation polymorphism method until 2009 (10) and with the peptide nucleic acid-locked nucleic acid polymerase chain reaction clamp method from 2010 onward. The HER2 and BRAF mutations were also examined using PCR-single-strand conformation polymorphism $(11,12)$. The KRAS mutation was investigated using a modified mutagenic PCR restriction fragment length polymorphism technique (10). $A L K$ fusion was detected via reverse transcription PCR and fluorescence in situ hybridization (FISH) until 2009 and via immunohistochemistry and FISH from 2010 onward $(13,14)$. ROS1 fusion was detected via FISH using a ROS1 Dual Color Break Apart Probe (Vysis LSI/ Abbott Laboratories, Chicago, IL, USA) according to the manufacturer's instructions (15). RET fusion was also detected via FISH using the Kreatech RET (10q11) Break FISH probe (Leica Biosystems, Wetzlar, Germany) and RET Split Dual Color FISH probe (GSP Lab., Tokyo, Japan) according to the manufacturer's instructions (15).

\section{Statistics}

The $\chi^{2}$ and Fisher's exact tests were used to analyze categorical data. Survival rates were calculated using the Kaplan-Meier method, and differences in overall survival (OS) and disease-free survival (DFS) were analyzed using the log-rank test. Univariate and multivariate analyses were performed using the Cox's proportional hazards model. A $\mathrm{P}$ value $<0.05$ was considered statistically significant. Data analysis and graph-plotting were performed using the JMP statistical software package version 12 (SAS Institute, Cary, NC, USA).

\section{Results}

\section{Patient and tumor characteristics}

The clinicopathological characteristics of the study population are shown in Table 1; there were 514 (48.6\%) men and $543(51.4 \%)$ women with a mean age of $66.2 \pm 9.8$ years at diagnosis. The mean tumor size was $23.9 \pm 14.4 \mathrm{~mm}$; and $52.9 \%$ of the patients were smokers (including current and former smokers). Moreover, $2.0 \%$ of 
Table 1 Association between cribriform pattern and clinicopathological features

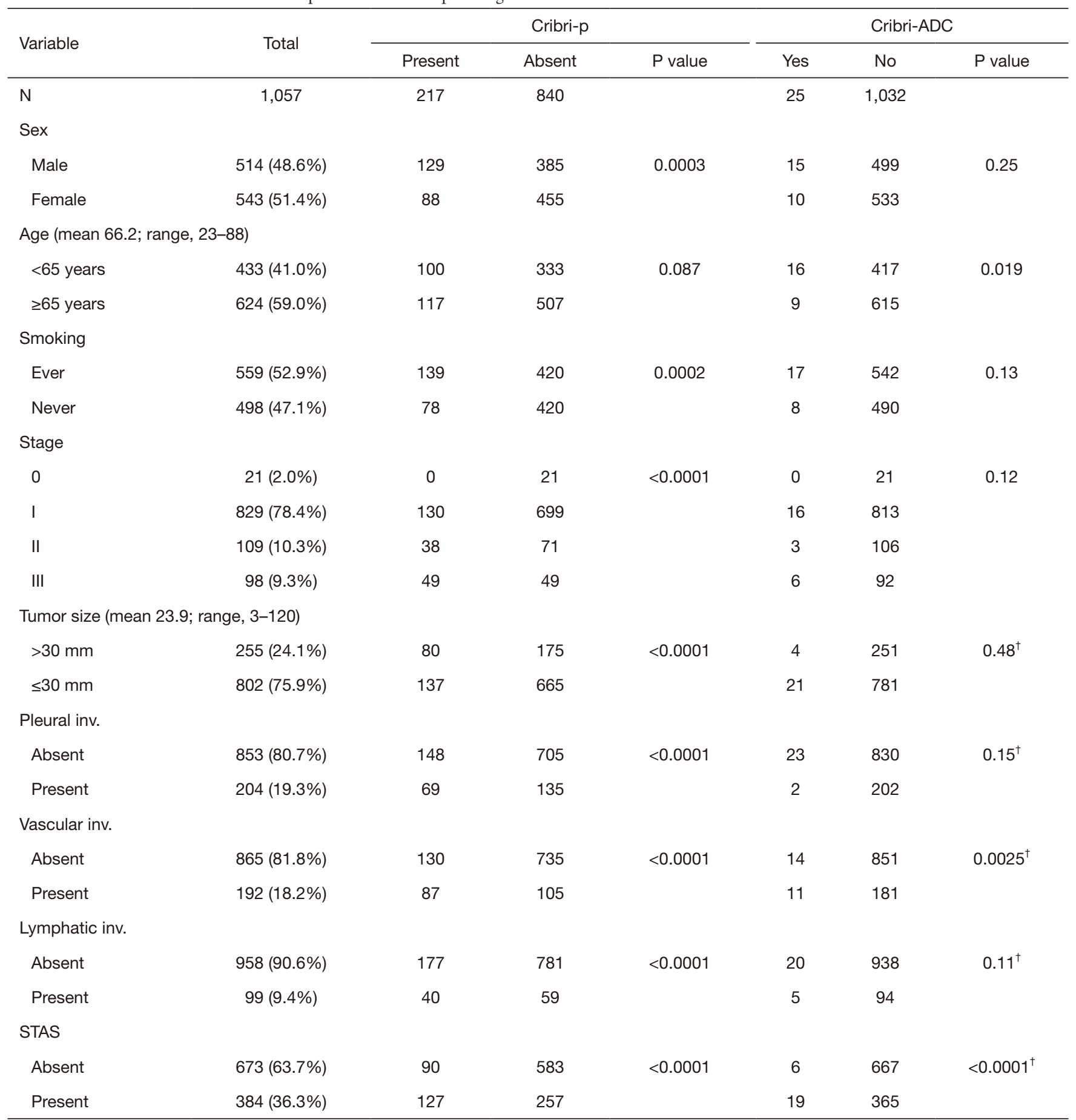

${ }^{\dagger}$, Fisher's exact test. Cribri-p, cribriform pattern; Cribri-ADC, cribriform predominant adenocarcinoma; STAS, spread through air spaces; inv., invasion; MP, micropapillary. 
patients had stage 0 disease, $78.4 \%$ had stage I, $10.3 \%$ had stage II, and $9.3 \%$ had stage III. The proportion of stage I patients in our cohort was much higher and the proportion of smokers in our cohort was lower than that in the European cohort (6). However, the proportions were comparable to the other data from Japan or Asian countries $(8,9)$. One hundred seventy-nine patients (16.9\%) died during follow-up and 231 $(21.9 \%)$ relapsed. The mean follow-up time at the end-point of analysis was $61.6 \pm 35.7$ months.

\section{Frequency of tumors with Cribri-p/Cribri-ADC and their associated clinicopathological features}

Cribri-p was present in 217 of 1,057 patients (20.5\%), and the median proportion of this component was $20.3 \%$ (range, $5-90 \%$ ) (Figure S1A). The median proportion of tumor with the fused-gland and sieve-like patterns were $5.5 \%$ (range, 5-30\%) and $17.9 \%$ (range, 5-90\%), respectively (Figure S1B,C). Tumors with only fused-gland patterns and those with both fused-gland and sieve-like patterns were observed in $54(5.1 \%)$ and $163(15.4 \%)$ patients, respectively (Table $\mathrm{S} 1$ ). In the analysis of detailed cribriform pattern, tumor with sieve-like pattern was frequently observed in tumors with solid pattern $(\mathrm{P}<0.0001)$. There were 25 patients with Cribri-ADC (2.4\%) after adjusting for the number of invasive adenocarcinoma subtypes based on the current WHO classification (Figure S2A,B).

The relationships between Cribri-p and certain clinicopathological features are listed in Table 1. Cribri-p was observed more frequently in men $(\mathrm{P}=0.0003)$ and smokers $(\mathrm{P}=0.0002)$. It was most frequent in patients with stage III disease $(49,50.0 \%)$ followed by stage II (71, $34.9 \%)$, whereas it was not frequently associated with stage I (130 patients, $15.7 \%)$. There was no statistically significant relationship between the presence of Cribri-p and age. Cribri-p was also associated with a larger tumor size $(\mathrm{P}<0.0001)$, pleural invasion $(\mathrm{P}<0.0001)$, vascular invasion $(\mathrm{P}<0.0001)$, lymphatic invasion $(\mathrm{P}<0.0001)$, and spread through air spaces (STAS) $(\mathrm{P}<0.0001)$. Cribri-p was also most frequently observed in solid adenocarcinomas (51.3\%) followed by adjusted acinar adenocarcinomas $(32.7 \%)$, whereas it was rare in lepidic adenocarcinomas (2.8\%) (Figure S2C).

Cribri-ADC was frequently observed in younger patients $(\mathrm{P}=0.019)$ and was also associated with vascular invasion $(\mathrm{P}=0.0025)$, solid pattern $(\mathrm{P}<0.0001)$, and STAS $(\mathrm{P}<0.0001)$ (Table 1). However, Cribri-ADC was not associated with sex, smoking status, stage, tumor size, or the presence of pleural invasion.

\section{Associations between Cribri-p/Cribri-ADC and gene alteration}

Among the 1057 L-ADCs, 497, 221, 145, 228, 393, 262, and 236 tumors were assessed for EGFR, KRAS, HER2, $B R A F$ mutations, and ALK, RET, and ROS1 fusions, respectively. Cribri-p was significantly associated with wildtype $E G F R, K R A S$ mutations, and $A L K$ rearrangement $(\mathrm{P}=0.0041, \mathrm{P}=0.023$, and $\mathrm{P}=0.0002$, respectively) but was not significantly associated with HER2 mutations, BRAF mutations, RET rearrangement, or ROS1 rearrangement (Table 2). Cribri-ADC was only associated with tumors exhibiting ALK rearrangement $(\mathrm{P}=0.012)$.

\section{Association between Cribri-p/Cribri-ADC and prognosis}

Cribri-p was associated with significantly higher risks of death and recurrence in patients with all stages (relative risk 2.5; 95\% confidence interval 1.9-3.2, $\mathrm{P}<0.0001)$ and in those with stage I (relative risk $2.1 ; 95 \%$ confidence interval 1.3-3.3, $\mathrm{P}=0.0044)$, but not in those with stages II or III (Table S2). Next, we investigated the prognostic significance of Cribri-p in the acinar-, papillary-, solid-, and micropapillary-ADC. Regarding papillary adenocarcinoma, patients with Cribri-p had significantly worse prognoses than did those without (death: $\mathrm{P}=0.0096$, recurrence: $\mathrm{P}=0.0016$ ) (Table S2). As for micropapillary adenocarcinoma, patients with Cribri-p had significantly higher risks of recurrence than did those without $(\mathrm{P}=0.024)$ (Table $\mathrm{S} 2)$. There were no statistically significant differences in terms of the risk of death and recurrence between patients with acinar and solid adenocarcinoma who had Cribri-p and those who did not. We used factors that were found to be significant in the univariate analysis (data not shown) for the multivariate analysis; Cribri-p was found to not be an independent prognostic factor, whereas a micropapillary pattern was an independent predictor of recurrence while a solid pattern was an independent predictor of both recurrence and death (Table S3, Trial 1).

L-ADC was reclassified into seven groups based on the predominant pattern: lepidic, acinar, papillary, solid, micropapillary, variant, and cribriform (Figure S2B). The DFS curves of patients with Cribri-ADC were identical to those of patients with solid adenocarcinoma; furthermore, the DFS of patients with Cribri-ADC was shorter than that of patients with lepidic adenocarcinoma and papillary 
Table 2 Association between cribriform pattern and gene alterations

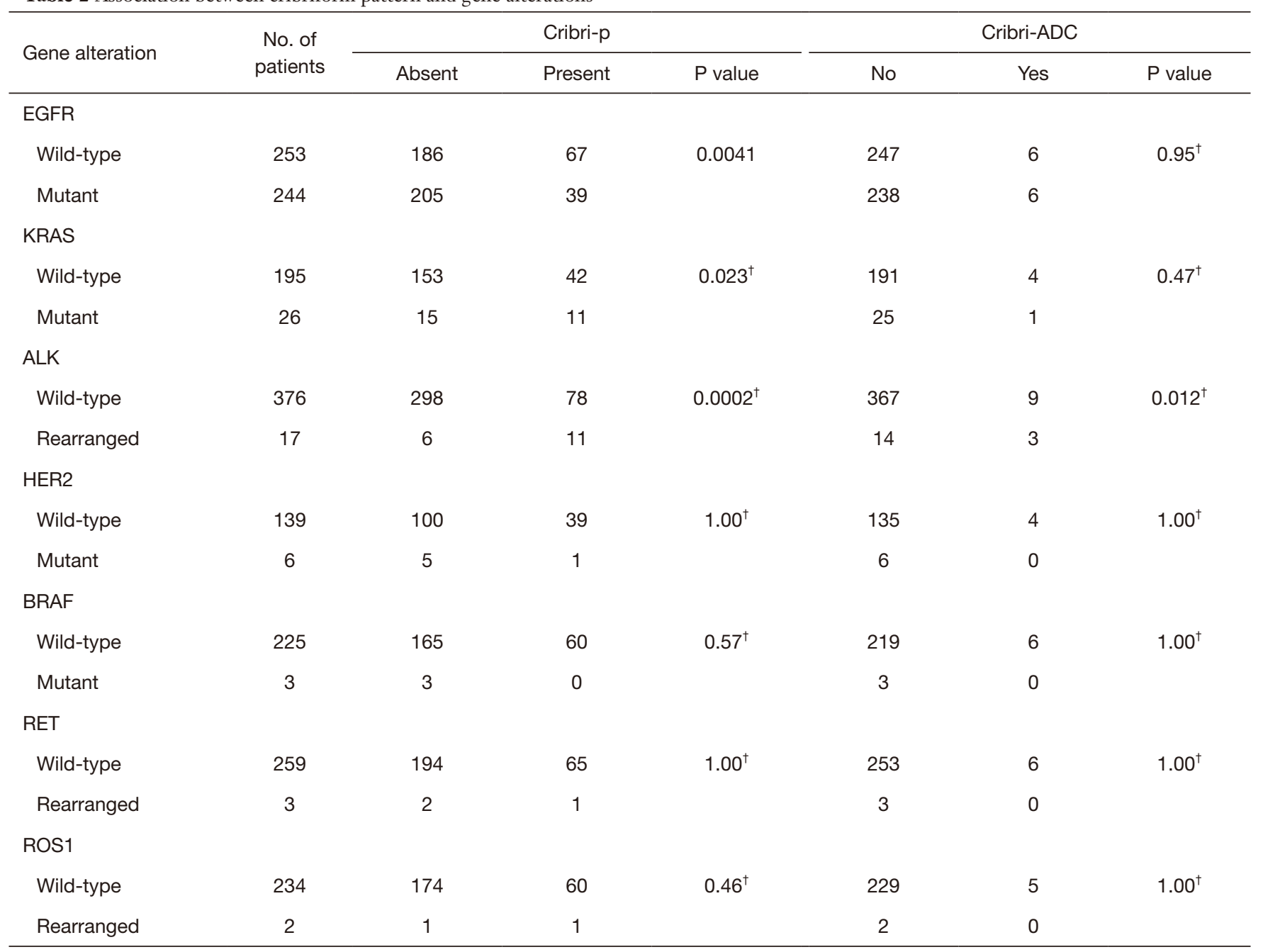

${ }^{\dagger}$, Fisher's exact test. Cribri-p, cribriform pattern; Cribri-ADC, cribriform predominant adenocarcinoma.
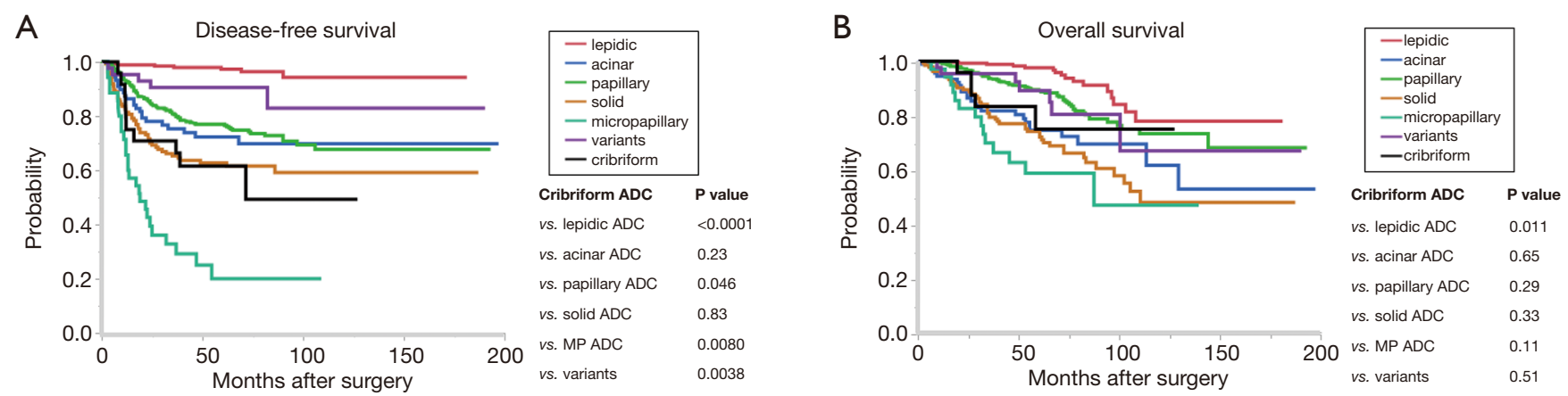

Figure 2 Association between adenocarcinoma (ADC) subtype and disease-free survival (DFS) and overall survival (OS). (A) The DFS of patients with cribriform $\mathrm{ADC}$ is shorter than that of patients with lepidic $\mathrm{ADC}(\mathrm{P}<0.0001)$ and those with papillary ADC $(\mathrm{P}=0.046)$, while the survival curve of patients with cribriform ADC was similar to that of patients with solid ADC; (B) the OS of patients with cribriform ADC was relatively favorable compared to that of patients with solid and micropapillary adenocarcinoma. MP, micropapillary. 
Table 3 Status after initial resection of cribriform adenocarcinoma in patients with recurrence

\begin{tabular}{|c|c|c|c|c|c|c|c|c|c|c|}
\hline No. & Status & Stage (8th) & $\begin{array}{l}\text { Last follow- } \\
\text { up (months) }\end{array}$ & $\begin{array}{c}\text { Recurrent } \\
\text { months after } \\
\text { surgery }\end{array}$ & $\begin{array}{c}\text { Recurrence or } \\
\text { metastasis status }\end{array}$ & $\begin{array}{c}\text { EGFR } \\
\text { mutations }\end{array}$ & $\begin{array}{c}\text { ALK } \\
\text { rearrangement }\end{array}$ & Tx-TKls & Tx-Chemo & Tx-Rad \\
\hline 2 & DOD & 3A (T1bN2M0) & 26 & 8 & CNS & NA & NA & No & Yes & Yes \\
\hline 3 & DOD & 2B (T1bN1M0) & 58 & 11.5 & CNS & Yes & NA & Yes & Yes & Yes \\
\hline 5 & DOD & 1A2 (T1bNOM0) & 26 & 11.8 & CNS & Yes & NA & Yes & No & No \\
\hline 6 & AWD & 2B (T1bN1M0) & 62.5 & 36.9 & $\begin{array}{l}\text { Mediastinal and } \\
\text { axillary LN }\end{array}$ & Yes & No & Yes & Yes & No \\
\hline 10 & AWD & 3A (T1cN2M0) & 44 & 38.8 & $\begin{array}{l}\text { Mediastinal LN, lung, } \\
\text { bone }\end{array}$ & Yes & No & Yes & Yes & Yes \\
\hline
\end{tabular}

AWD, alive with disease; DOD, dead of disease; CNS, central nervous system; LN, lymph node; NA, not available; Tx-TKIs, treatment with tyrosine kinase inhibitors; Tx-Chemo, platinum-base chemotherapy; Tx-Rad, radiation therapy.

adenocarcinoma, and was longer than that of patients with micropapillary adenocarcinoma $(\mathrm{P}<0.0001, \mathrm{P}=0.046$, and $\mathrm{P}=0.0080$, respectively) (Figure $2 A$ ). The OS curve of patients with Cribri-ADC was located between that of patients with papillary and acinar adenocarcinoma (Figure $2 B$ ). In multivariate analysis, Cribri-ADC was not an independent prognostic factor of either recurrence or death, whereas age ( $>65 v s . \leq 65$ years), stage (II $v s$. I and III $v s$. I), lymphatic invasion, and pleural invasion were independent predictors of both recurrence and death (Table S3, Trial 2). Table 3 shows the statuses of 10 patients with CribriADC with recurrence after resection. EGFR mutations or $A L K$ rearrangements were detected in all six patients who were alive with disease, those of whom achieved long survival (median, 64.6 months; range, 37.4-113 months) following treatment with tyrosine kinase inhibitors (TKIs) like gefitinib, erlotinib, or crizotinib. Among the four deceased patients, EGFR mutations were detected in two; these patients were also treated with TKIs and died 58 and 26 months after initial resection, respectively.

As for detail of Cribri-p and prognosis, the 5-year DFS rate of patients exhibiting tumors with only the fusedgland pattern tended to be lower than that of patients having tumors without Cribri-p but higher than that of patients having tumors with sieve-like pattern, although these differences were not significant $(\mathrm{P}=0.10$ and $\mathrm{P}=0.053$, respectively) (Figure S3A). Moreover, the five-year OS rate of patients with only fused-gland pattern tumors were significantly higher than that of those with sieve-like pattern tumors $(\mathrm{P}=0.037)$, although there was no significant different in OS between those with fused-gland pattern tumors and those without Cribri-p tumors $(\mathrm{P}=0.364)$ (Figure S3B).

\section{Discussion}

We found that 25 of our patients (2.4\%) had Cribri$\mathrm{ADC}$, and that this pattern was associated with male sex, lymphovascular invasion, and STAS. Patients with CribriADC had relatively worse DFS rates than did those with acinar adenocarcinoma but had similar rates to those of patients with solid-ADC. The OS curve of patients with Cribri-ADC was located between that of patients with papillary and acinar adenocarcinoma. Most patients with recurrent tumors harbored druggable gene alterations and achieved relatively long-term survival following treatment with TKIs. Our results indicate that Cribri-ADC is the unfavorable lung adenocarcinoma subgroup in terms of prognosis, but that it can be controlled with the appropriate TKIs. 
A summary of previous large-scale studies that investigated Cribri-ADC is shown in Table 4. In six previous studies (4-9), the frequency of Cribri-ADC was found to be $4.2-12 \%$. The DFS of patients with Cribri-ADC tended to be worse than that of patients with acinar L-ADC but comparable to that of patients with solid ADC in five of the studies (4,5,7-9). Our current study demonstrated that patients with Cribri-ADC had DFS rates that were relatively worse than those with acinar L-ADC but comparable to those with solid-ADC; thus, our results are consistent with those from previous studies (4-9). Based on the evidence that Cribri-ADC tends to recur, we agree with the notion that Cribri-ADC should be considered a new tumor subtype derived from acinar-ADC.

With respect to OS, three previous studies (7-9) demonstrated that patients with Cribri-ADC have relatively poor outcomes compared to patients with solid ADC. Warth et al. reported that the OS rates of patients with Cribri-ADC was in between those of patients with acinar ADC and solid ADC (6); our data were consistent with theirs. They suggested that the natural course of Cribri$\mathrm{ADC}$ is aggressive but that these tumors might respond better to chemotherapy administered after relapse than would tumors of the other patterns. In the current study, eight of 10 patients who experienced recurrence harbored $E G F R$ mutations or $A L K$ rearrangements (data were not available for the other two), and six were alive with disease following treatment with TKIs.

In our study, Cribri-p was observed in $20.1 \%$ of L-ADCs and was associated with several clinicopathologic factors; patients of all stages, stage I, and papillary adenocarcinoma subtype with Cribri-p had significantly higher risks of death or recurrence than did those without. Regarding the significance of Cribri-p as a minor component, Kadota et al. reported that the 'recurrence-free probability' among patients with predominantly acinar tumors exhibiting $\geq 10 \%$ Cribri-p was significantly lower than that among patients with tumors exhibiting $<10 \%$ Cribri-p (4). Moreover, they also demonstrated that the presence of Cribri-p did not correlate with the risk of recurrence among patients with papillary or solid predominant tumors. In our study, we found that the DFS and OS rates of patients with papillarypredominant adenocarcinomas who had $\geq 5 \%$ Cribri-p were significantly lower, and that their recurrence rates were significantly higher than those of patients with $<5 \%$ Cribri-p. In contrast, there were no significant differences in the risks of death and recurrence between patients with acinar and solid adenocarcinomas with $\geq 5 \%$ versus those with $<5 \%$ Cribri-p. This discrepancy may be owing to the differences in the thresholds used to determine the presence of Cribri-p. However, we recommend that the presence of Cribri-p be reported as a potential prognostic variable even if other predominant subtypes are noted.

Regarding the association between Cribri-p and gene alterations, earlier studies found that tumors with $A L K$ rearrangements tended to exhibit Cribri-p $(6,7,16,17)$, while others found no such association $(8,9)$. As such, a clear link between these two occurrences has not been established. In our study, the presence of Cribri-p and Cribri-ADC was associated with $A L K$ rearrangement. The discrepancy between the studies may be caused by differences in the thresholds and/or methodologies used for identifying gene alteration; hence, the relationship between gene alterations and Cribri-p ought to be investigated using uniform methods such as next-generation sequencing.

There were some limitations in this study, the most notable of which was the inter-observer agreement. Although we subclassified Cribri-p from acinar-pattern tumors after careful discussion, we noted some difficulty in distinguishing simple acinar glands from fused-glands because most were continuously present in the same regions. However, we consider that we can overcome interobserver disagreement by training to recognize the pattern carefully because our concordance rate was not very low when analyzing the selected 100 cases (data not shown). In contrast, if the Cribri-ADC is recognized as the sixth histological subtype of L-ADC, it will potentially be more difficult to reach interobserver agreement than it would be with the current scheme. Deep learning techniques may be promising to overcome this difficulty $(18,19)$.

In addition, we also consider classification by tumor grading to be more optimal than determining the tumor subtype for predicting the prognosis (20). While some grading systems have been proposed, additional multicenter validation studies are needed. A second limitation was that some investigators set their Cribri-p cut-off values at $5 \%$ and others at $10 \%$; we set ours at $5 \%$ because the percentage of each histological component is recorded in $5 \%$ increments per the 2015 WHO classification recommendations (3). As such, Cribri-p should be more strictly identified because the presence of other subtypes with Cribri-p appear to be associated with relatively poorer prognoses; this also requires future validation. 


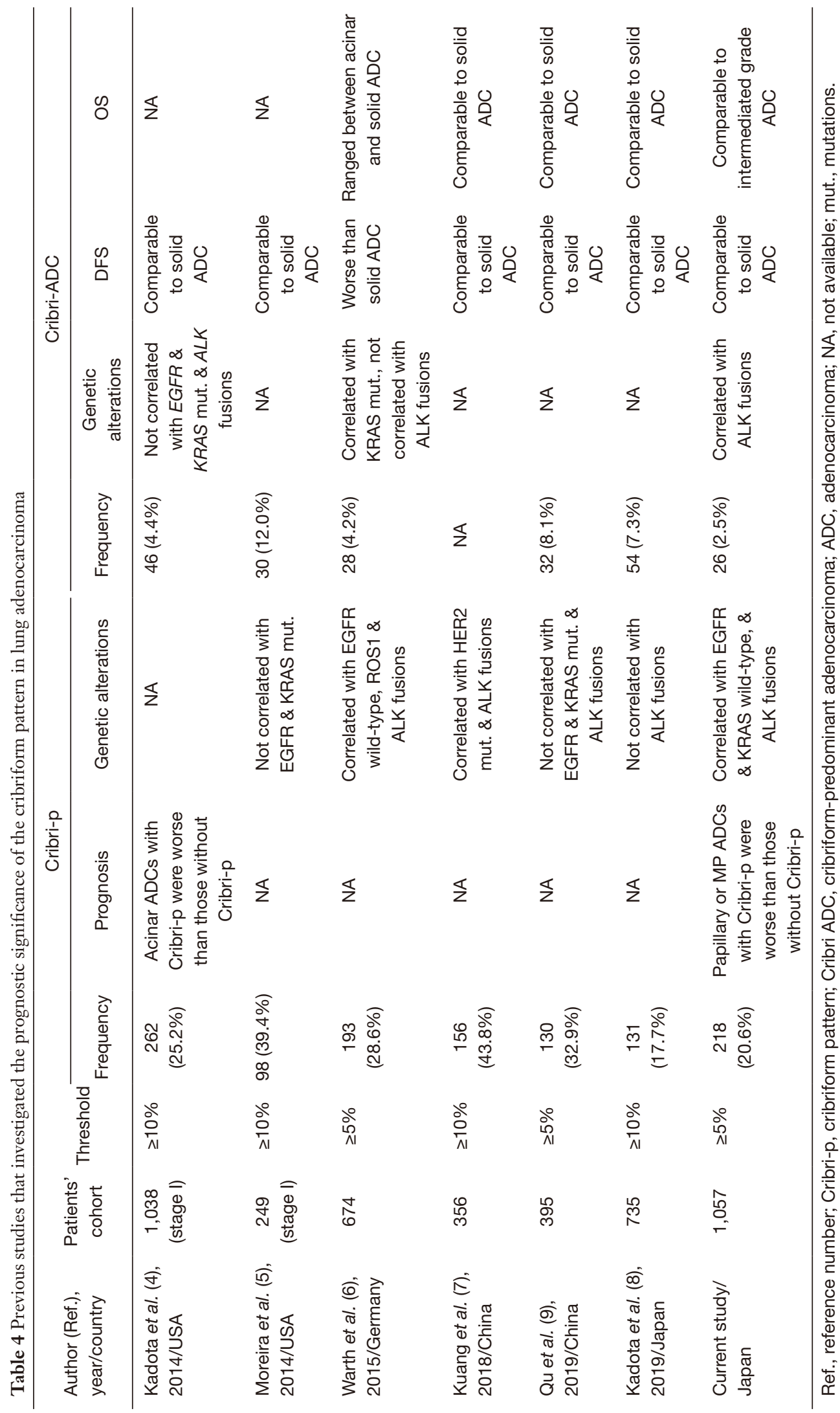




\section{Conclusions}

Our evaluation of the clinical significance of Cribri-ADC revealed that this pattern portends a poor prognosis with respect to recurrence, which is consistent with data from previous studies. However, most patients with recurrent tumors harbor druggable genetic alterations, such as EGFR mutations and $A L K$ rearrangements, and are therefore responsive to TKIs. Given these results, we propose that TKIs should be added to the postoperative adjuvant therapy regimens administered to such patients. Furthermore, our findings suggest that Cribri-p should be recognized as a poor prognostic factor even when it is not a major tumor component.

\section{Acknowledgments}

Funding: This work was supported in part by JSPS KAKENHI Grant Number 17K08740.

\section{Footnote}

Reporting Checklist: The authors have completed the STROBE reporting checklist. Available at http://dx.doi. org/10.21037/tlcr-20-612

Peer Review File: Available at http://dx.doi.org/10.21037/ tlcr-20-612

Conflicts of Interest: All authors have completed the ICMJE uniform disclosure form (http://dx.doi.org/10.21037/tlcr20-612). AY reports that grants from JSPS KAKENHI, during the conduct of the study. The other authors have no conflicts of interest to declare.

Ethical Statement: The authors are accountable for all aspects of the work in ensuring that questions related to the accuracy or integrity of any part of the work are appropriately investigated and resolved. The requirement for informed consent was waived owing to the retrospective nature of the study and all patients were provided the means to opt out of this study. The study was conducted in accordance with the Declaration of Helsinki (as revised in 2013) and the study protocol was approved by the Kyoto University Hospital ethics committee (R1158-2).

Open Access Statement: This is an Open Access article distributed in accordance with the Creative Commons
Attribution-NonCommercial-NoDerivs 4.0 International License (CC BY-NC-ND 4.0), which permits the noncommercial replication and distribution of the article with the strict proviso that no changes or edits are made and the original work is properly cited (including links to both the formal publication through the relevant DOI and the license). See: https://creativecommons.org/licenses/by-nc-nd/4.0/.

\section{References}

1. Ferlay J, Ervik M, Lam F, et al. Global Cancer Observatory: Cancer Today. Lyon, France: International Agency for Research on Cancer, [cited 06 February 2020] Available online: https://gco.iarc.fr/today

2. Siegel RL, Miller KD, Jemal A. Cancer statistics, 2019. CA Cancer J Clin 2019;69:7-34.

3. Travis WD, Brambilla E, Burke A, et al. WHO Classification of Tumours of the Lung, Pleura, Thymus and Heart. 4th Edition. Lyon: International Agency for Research on Cancer, 2015.

4. Kadota K, Yeh YC, Sima CS, et al. The cribriform pattern identifies a subset of acinar predominant tumors with poor prognosis in patients with stage I lung adenocarcinoma: a conceptual proposal to classify cribriform predominant tumors as a distinct histologic subtype. Mod Pathol 2014;27:690-700.

5. Moreira AL, Joubert P, Downey RJ, et al. Cribriform and fused glands are patterns of high-grade pulmonary adenocarcinoma. Hum Pathol 2014;45:213-20.

6. Warth A, Muley T, Kossakowski C, et al. Prognostic impact and clinicopathological correlations of the cribriform pattern in pulmonary adenocarcinoma. $\mathrm{J}$ Thorac Oncol 2015;10:638-44.

7. Kuang M, Shen X, Yuan C, et al. Clinical Significance of Complex Glandular Patterns in Lung Adenocarcinoma: Clinicopathologic and Molecular Study in a Large Series of Cases. Am J Clin Pathol 2018;150:65-73.

8. Kadota K, Kushida Y, Kagawa S, et al. Cribriform Subtype is an Independent Predictor of Recurrence and Survival After Adjustment for the Eighth Edition of TNM Staging System in Patients With Resected Lung Adenocarcinoma. J Thorac Oncol 2019;14:245-54.

9. Qu Y, Lin H, Zhang C, et al. Cribriform pattern in lung invasive adenocarcinoma correlates with poor prognosis in a Chinese cohort. Pathol Res Pract 2019;215:347-53.

10. Sonobe M, Kobayashi M, Ishikawa M, et al. Impact of KRAS and EGFR gene mutations on recurrence and survival in patients with surgically resected lung 
adenocarcinomas. Ann Surg Oncol 2012;19: S347-354.

11. Sonobe M, Manabe T, Wada H, et al. Lung adenocarcinoma harboring mutations in the ERBB2 kinase domain. J Mol Diagn 2006;8:351-6.

12. Kobayashi M, Sonobe M, Takahashi T, et al. Clinical significance of BRAF gene mutations in patients with nonsmall cell lung cancer. Anticancer Res 2011;31:4619-23.

13. Takahashi T, Sonobe M, Kobayashi M, et al. Clinicopathologic features of non-small-cell lung cancer with EML4-ALK fusion gene. Ann Surg Oncol 2010;17:889-97.

14. Nakajima N, Yoshizawa A, Kondo K, et al. Evaluating the effectiveness of RNA in-situ hybridization for detecting lung adenocarcinoma with anaplastic lymphoma kinase rearrangement. Histopathology 2017;71:143-9.

15. Rokutan-Kurata M, Yoshizawa A, Sumiyoshi S, et al. Lung Adenocarcinoma With MUC4 Expression Is Associated With Smoking Status, HER2 Protein Expression, and Poor Prognosis: Clinicopathologic Analysis of 338 Cases. Clin Lung Cancer 2017;18:e273-e281.

Cite this article as: Nakajima N, Yoshizawa A, Rokutan-Kurata M, Noguchi M, Teramoto Y, Sumiyoshi S, Kondo K, Sonobe M, Hamaji M, Menju T, Date H, Haga H. Prognostic significance of cribriform adenocarcinoma of the lung: validation analysis of 1,057 Japanese patients with resected lung adenocarcinoma and a review of the literature. Transl Lung Cancer Res 2021;10(1):117-127. doi: 10.21037/tlcr-20-612
16. Inamura $\mathrm{K}$, Takeuchi $\mathrm{K}$, Togashi $\mathrm{Y}$, et al. EML4-ALK lung cancers are characterized by rare other mutations, a TTF1 cell lineage, an acinar histology, and young onset. Mod Pathol 2009;22:508-15.

17. Yoshida A, Tsuta K, Nakamura H, et al. Comprehensive histologic analysis of ALK-rearranged lung carcinomas. Am J Surg Pathol 2011;35:1226-34.

18. Wei JW, Tafe LJ, Linnik YA, et al. Pathologist-level classification of histologic patterns on resected lung adenocarcinoma slides with deep neural networks. Sci Rep 2019;9:3358

19. Gertych A, Swiderska-Chadaj Z, Ma Z, et al. Convolutional neural networks can accurately distinguish four histologic growth patterns of lung adenocarcinoma in digital slides. Sci Rep 2019;9:1483.

20. Sica G, Yoshizawa A, Sima CS, et al. A grading system of lung adenocarcinomas based on histologic pattern is predictive of disease recurrence in stage I tumors. Am J Surg Pathol 2010;34:1155-62. 


\section{Supplementary}

A

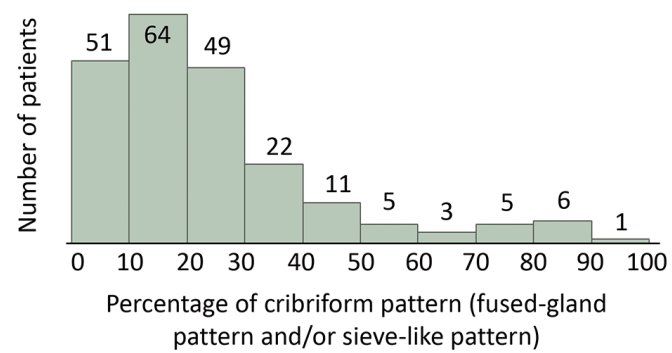

C

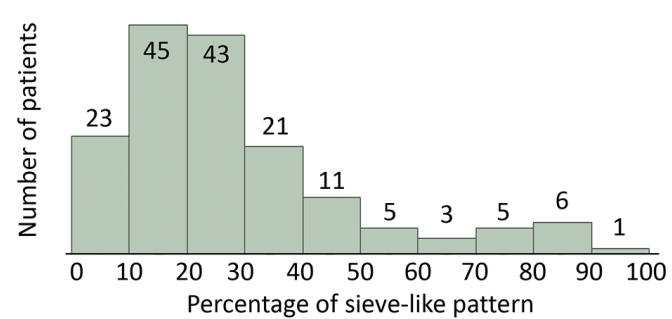

B

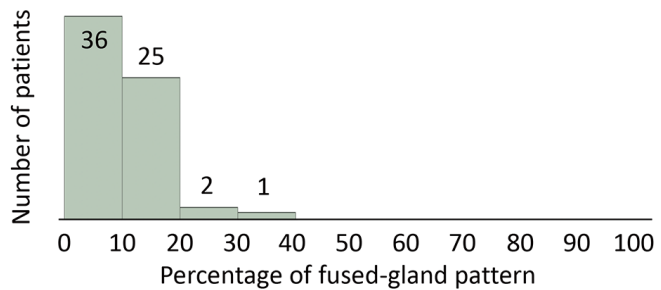

Figure S1 Histograms showing the proportions of cribriform patterns among patients with lung adenocarcinoma. (A) Percentages of cribriform patterns overall (i.e., combined fused-gland and sieve-like patterns); (B) percentages of fused-gland patterns; (C) percentages of sieve-like patterns. 
Table S1 Comparison among detailed cribriform patterns

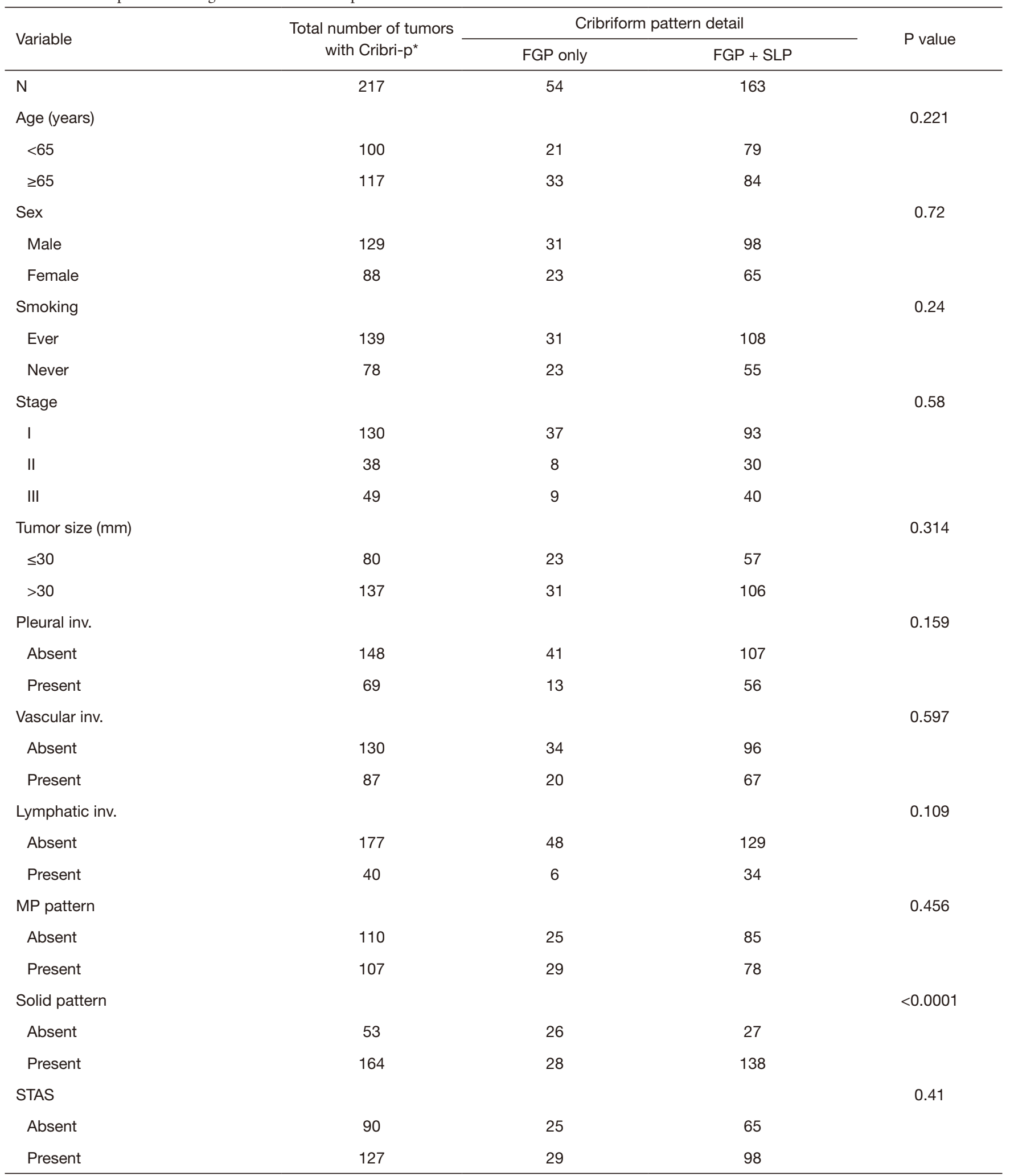

*, Cribri-p includes fused-gland pattern and/or sieve-like pattern. inv. invasion; MP p, micropapillary pattern; STAS, spread through air spaces; Cribri-p, cribriform pattern; FGP, fused-glands pattern; SLP, sieve-like pattern. 
A

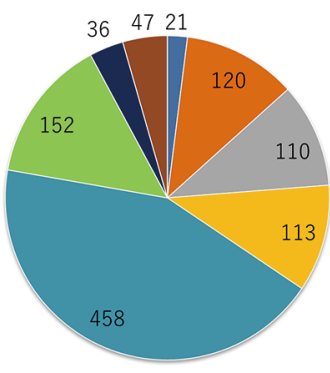

B

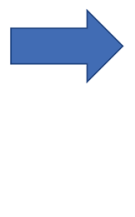

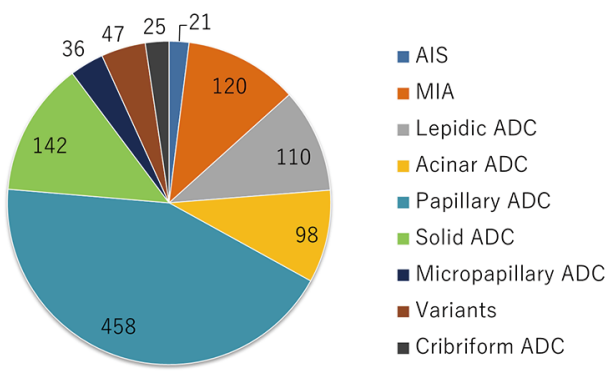

C

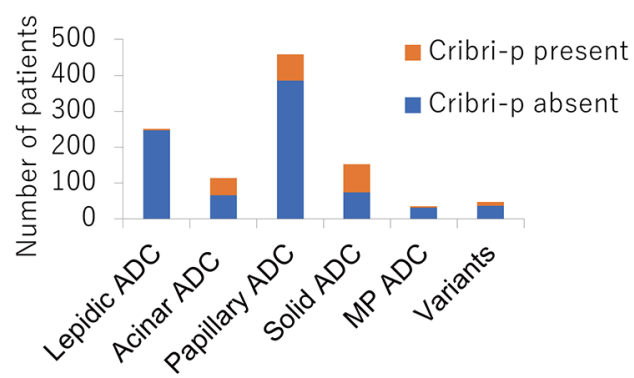

Figure S2 Association between the cribriform pattern and other histological patterns. (A) Proportion of histological subtype based on the 2015 World Health Organization classification; (B) proportion of histological subtypes when considering cribriform ADC; (C) cribriform pattern was the most frequently observed in solid ADCs (51.3\%) followed by adjusted acinar ADCs (32.7\%), whereas it was rare in lepidic ADCs (2.8\%). ADC, adenocarcinoma; AIS, adenocarcinoma in situ; Cribri-p, cribriform pattern; MIA, minimally invasive adenocarcinoma; MP, micropapillary.

Table S2 Univariate analysis of cribriform pattern and risk of death or recurrence per subgroup

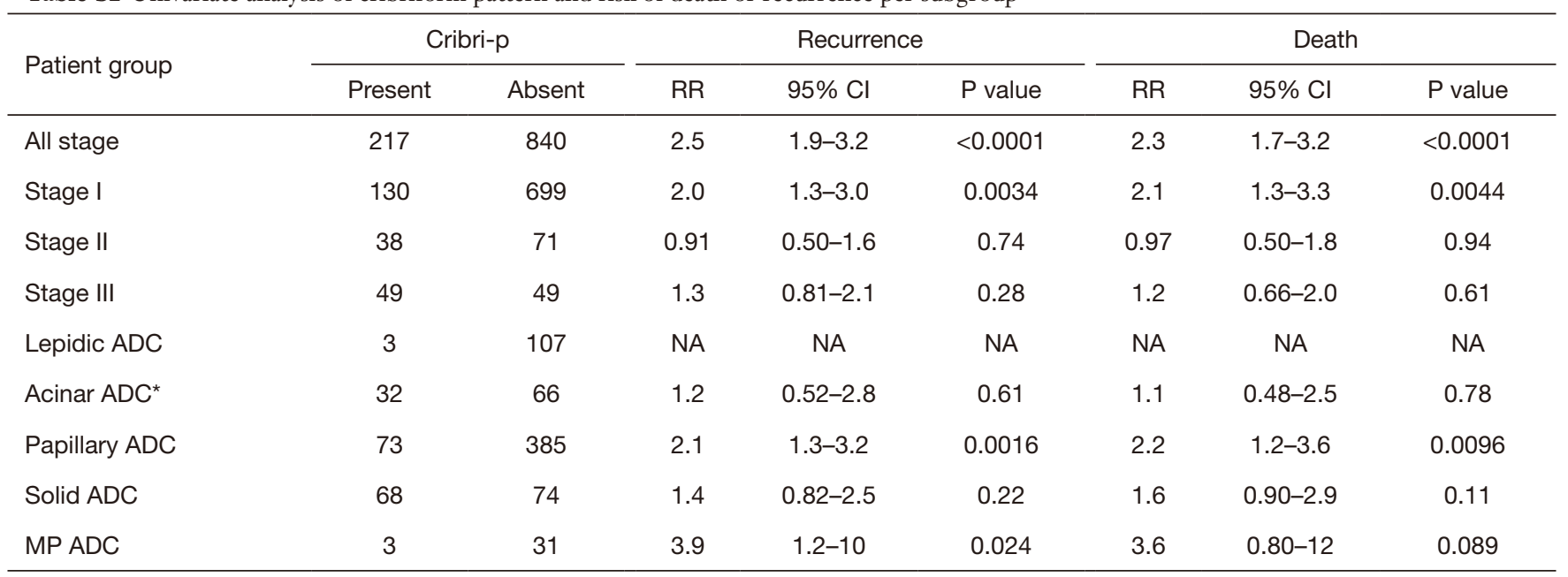

*, after extracting Cribri-p from 'acinar pattern' according to the 2015 World Health Organization classification. RR, relative risk; NA, not available; $\mathrm{Cl}$, confidence interval; $\mathrm{ADC}$, adenocarcinoma; Cribri-p, cribriform pattern; MP, micropapillary. 
Table S3 Multivariate analysis of cribriform pattern and cribriform adenocarcinoma

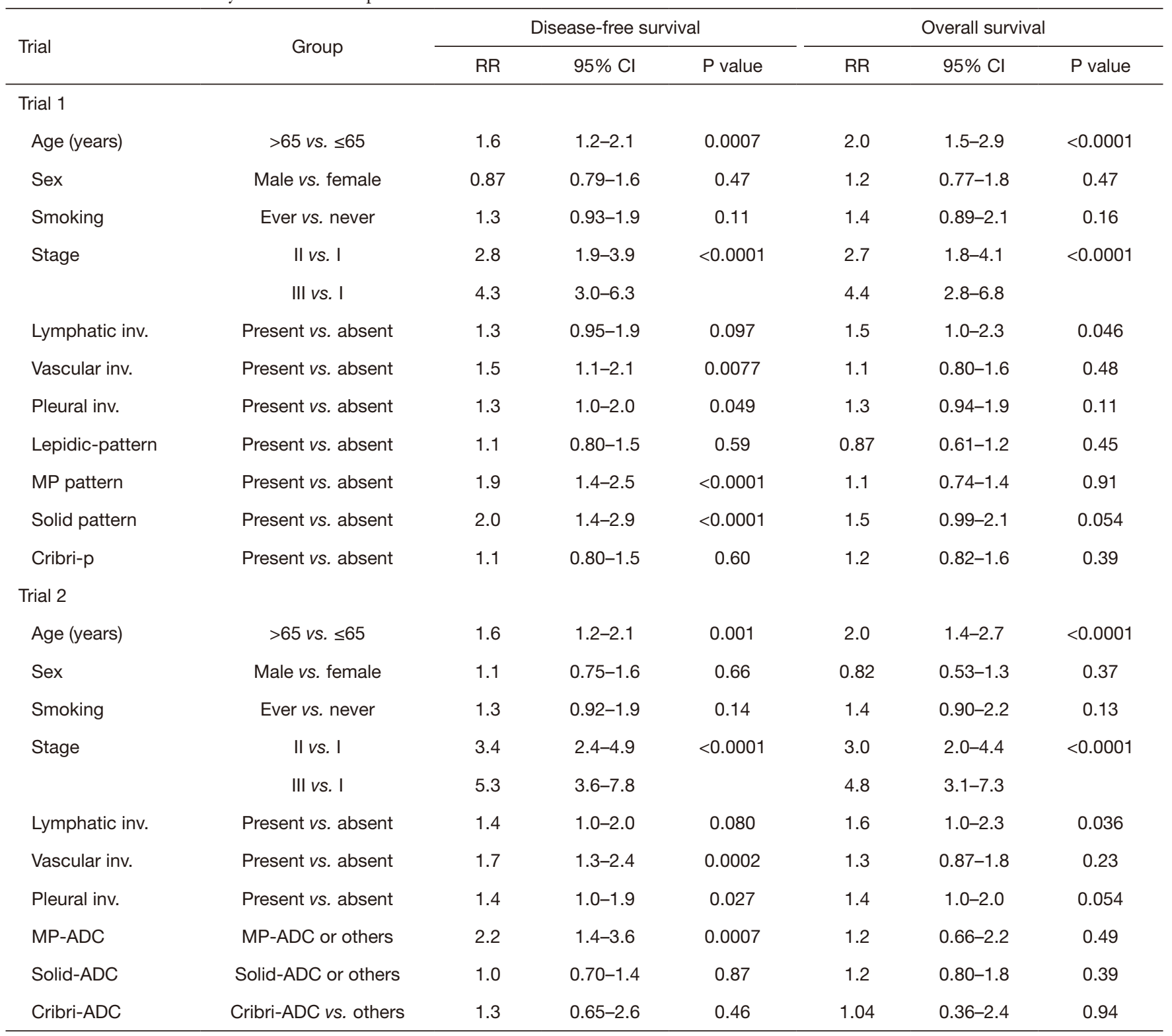

$\mathrm{RR}$, relative risk; $\mathrm{Cl}$, confidence interval; Cribri-p, cribriform pattern; inv., invasion; MP, micropapillary; MP-ADC, micropapillary adenocarcinoma; Solid-ADC, solid adenocarcinoma; Cribri-ADC, cribriform adenocarcinoma. 

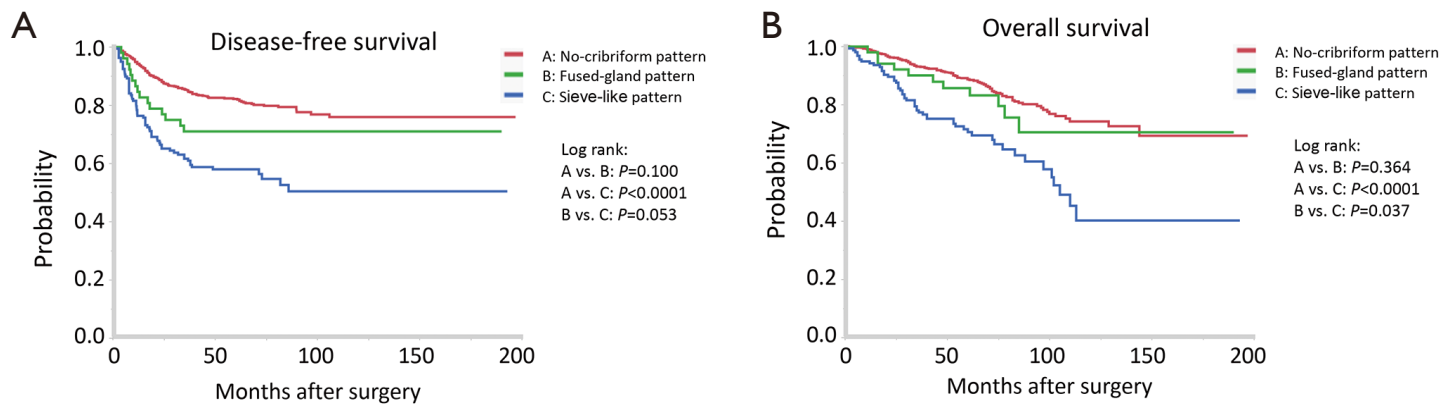

Figure S3 Comparison of the prognostic impacts of the fused-gland versus sieve-like patterns. (A) Disease-free survival of patients with fused-gland patterns tended to better than that of patients with sieve-like patterns, although the difference was not significant; (B) overall survival of patients with only fused-gland patterns was significantly better than that of patients with sieve-like patterns. 